\title{
Emprendimiento y crecimiento económico: una visión desde la literatura y los principales indicadores internacionales
}

\author{
Gary Flor \\ Universidad Andina Simón Bolívar, Sede Ecuador \\ gaflor44@yahoo.es
}

Fecha de presentación: 18 de febrero de 2016 • Fecha de aceptación: 11 de julio de 2016

Artículo de investigación 


\section{Gary Flor}

\section{Resumen}

El artículo realiza un análisis crítico de los trabajos teóricos y empíricos sobre la relación entre el capital emprendedor y el crecimiento económico. Para ello se realiza una revisión de la literatura sobre emprendimiento entre 2004 y 2014, a partir de las preguntas de investigación sobre el impacto del capital emprendedor en el crecimiento económico y la conceptualización y modos de operacionalización del capital emprendedor, que toman como base diez indicadores internacionales. Concluye que el capital emprendedor tiene impacto positivo en el crecimiento económico, muy sensible a la diversidad, ambigüedad e insuficiencia de los distintos modos de operacionalizarlo.

Palabras clave: emprendimiento, capital emprendedor, crecimiento económico, indicadores.

JEL: C52 Evaluación y contraste de modelos; G12 Valoración de activos financieros.

\section{Abstract}

The article makes a critical analysis of the theoretical and empirical work on the relationship between entrepreneurial capital and economic growth. For this, a review of the entrepreneurship literature between 2004 and 2014 is carried out; based on the research questions on the impact of entrepreneurial capital on economic growth and the conceptualization and modes of operationalization of entrepreneurial capital, based on ten international indicators. The article suggests that entrepreneurial capital has a positive impact on economic growth, which is very sensitive to diversity, ambiguity and insufficiency of the different ways of operating it.

Keywords: entrepreneurship, entrepreneurial capital, economic growth, indicators.

JEL: C52 Evaluation and contrast of models; G12 Valuation of financial assets.

\section{Resumo}

O artigo realiza uma análise crítica dos trabalhos teóricos e empíricos sobre a relação entre o capital empreendedor e o crescimento econômico. Para isso, faz uma revisão da literatura sobre empreendimento entre 2004 e 2014 a partir dos questionamentos sobre o impacto do capital empreendedor no crescimento econômico e sobre a conceitualização e os modos de operacionalização do capital empreendedor, tomando como base dez indicadores internacionais. $\mathrm{O}$ artigo sugere que o capital empreendedor produz um impacto positivo no crescimento econômico, muito sensível à diversidade, à ambiguidade e à insuficiência dos diferentes modos de operacionalizá-lo.

Palavras-chave: Empreendimento, capital empreendedor, crescimento econômico, indicadores. JEL: C52 Avaliação, validação e seleção de modelos; G12 Valoração de ativos financeiros. 


\section{Introducción}

T 1 objetivo principal de este trabajo es efectuar un análisis crítico de los trabajos teóricos y empíricos sobre la relación entre el capital emprendedor y el crecimiento económico. Los antecedentes del capital emprendedor se remontan a Marshall (1916), que lo identificó con la capacidad organizativa como cuarto factor productivo, junto con la tierra, el capital físico y la mano de obra. El significado de este cuarto factor productivo y su relación con la teoría del crecimiento está muy bien especificado en la literatura (Holcombe 1998; Naudé 2013), pero en lo que se centra este trabajo es en el entendimiento actual del capital emprendedor -como la capacidad sistémica que tiene un país, región, ciudad, industria o sector para generar nuevas empresas o actividades emprendedoras (Audretsch y Monsen 2008)y su relación con el crecimiento económico, a partir del supuesto derivado de la teoría del desarrollo territorial endógeno, según la cual la densidad regional de emprendimientos se relaciona directamente con el nivel de desarrollo (Julién 2005). La relevancia del tema no es menor, por cuanto desde la crisis financiera de 2009, ocasionada por la burbuja inmobiliaria en Estados Unidos y Europa y su actual generalización a los países en desarrollo, el emprendimiento se ha empezado a contemplar como factor de amortiguación o resiliencia ante la crisis y propiamente como la solución a la salida de la recesión (Roudini 2012; Rüdiger, Peris-Ortiz y Blanco Gonzales 2014).

La metodología utilizada consistió en la revisión de la literatura teórica y empírica, revisión entendida de manera convencional como el método sistemático, explícito y reproducible para identificar, evaluar y sistematizar documentos relacionados que constan en publicaciones académicas y bases de datos relacionadas. Esta revisión de literatura dio lugar a la cualificación de la hipótesis que ha servido para dar respuesta tentativa a las preguntas que guiaron el desarrollo de la presente investigación: se considera que el capital 
emprendedor tiene impacto positivo en el crecimiento económico, pero dicho impacto es muy sensible a la diversidad, ambigüedad e insuficiencia de los distintos modos de operacionalizarlo.

Para responder a las preguntas de investigación sobre el impacto del capital emprendedor en el crecimiento económico y la conceptualización y modos de operacionalización del capital emprendedor (dimensiones, indicadores y sistemas para su medición) y contrastar así la hipótesis, se revisaron sistemáticamente los principales trabajos teóricos y empíricos de la literatura para el período 2004-2014 y los diez principales indicadores internacionales que aproximan el emprendimiento: ya sea de manera directa (Global Entrepreneurship Monitor, Global Entrepreneurship and Development Index, Índice de Condiciones Sistémicas para el Emprendimiento Dinámico, como índices compuestos; y Comparative Entrepreneurship Data for International Analysis, International Benchmark of Entrepreneurs y Eurostat Entrepreneurship Indicators Programme, como dashboards o tableros de datos); ya sea de manera indirecta (Índice Global de Competitividad, Doing Business y Global Innovation Index, como índices compuestos; y el World Bank Group Entrepreneurship Snapshots, como dashboard).

En la segunda sección se analiza la literatura existente sobre la relación entre el emprendimiento y el crecimiento económico, con algunas consideraciones sobre el estado de la cuestión en América Latina y Ecuador en particular. En la tercera sección se evalúan los principales índices compuestos y dashboards que aproximan directa o indirectamente el fenómeno del emprendimiento. En la cuarta sección se hace el balance de ventajas y desventajas de la aplicación de dichos indicadores para estudiar la relación entre emprendimiento y crecimiento económico y las políticas públicas de fomento emprendedor. El trabajo finaliza con las conclusiones y algunas consideraciones finales sobre la utilización de estos indicadores para la definición de políticas públicas relativas al fomento del emprendimiento en países en desarrollo.

\section{Análisis de estudios teóricos y empíricos}

La relación del capital emprendedor y el crecimiento económico es bidireccional. De hecho, los primeros estudios realizados por Audretsch y Keil- 
bach (2004), que permitieron introducir el concepto del capital emprendedor como una subcategoría del capital social, mostraron que existe una relación directa entre la creación de empresas y el crecimiento económico. A partir de una investigación efectuada en 327 zonas de Alemania se comprobó que las variaciones positivas del PIB de esas áreas tenían una influencia positiva en los niveles de emprendimiento, siendo dicha influencia mayor en zonas de alta tecnología y mayor densidad poblacional.

Para centrarse ya en la direccionalidad de este trabajo, la relación entre emprendimiento y crecimiento económico, otra investigación de Audretsch y Keilbach (2005), cuyo objetivo fue introducir el capital emprendedor como un concepto análogo al de capital social, ${ }^{1}$ planteó la hipótesis de que existen instrumentos para la definición de política económica en los países, entre ellos la promoción del emprendimiento y los incentivos a la creación de empresas, con el objetivo de lograr el crecimiento económico. Audretsch y otros (2006) propusieron luego el uso de tasas de variación de creación de empresas como indicador de la variable inobservable o latente del emprendimiento y, de esta manera, grandes dotaciones de capital emprendedor se verían reflejadas en tasas de creación de empresas elevadas.

A partir de estas bases conceptuales y metodológicas, la literatura sobre la relación entre el emprendimiento y el crecimiento económico se puede sistematizar teniendo en cuenta los canales a través de los cuales se materializa dicha relación -difusión del conocimiento, incremento de la productividad, generación de empleo- y cómo dicha relación está mediada por tipos de empresas/emprendimientos y niveles de desarrollo económico-empresarial.

Si se toman en cuenta los canales, los primeros aportes se centraron en la difusión del conocimiento. El trabajo de Acs y Vargas (2005) sobre el impacto del emprendimiento y las aglomeraciones en la variación de la tecnología a nivel nacional y su contribución al crecimiento económico en Estados Unidos, estableció que la difusión del conocimiento no solo depende de la

1. El capital emprendedor es un término diferente al de capital social (definido ampliamente como stock de confianza interpersonal y de los individuos en las instituciones): el primero incorpora otros elementos como la aceptación social y valoración del comportamiento empresarial, incluyendo sus aspectos actitudinales enfocados al riesgo y al fracaso en los negocios. Por ello, en el concepto del capital emprendedor inciden factores distintos y específicos, como algunos de naturaleza legal, institucional y social que, al igual que en el caso del capital social, hacen difícil su apropiada medición cuantitativa. 
cercanía geográfica de las regiones, sino también de las aglomeraciones industriales. Luego, Acs y otros (2005) mostraron que el emprendimiento prolongado tiene notable incidencia en el crecimiento económico, ya que este responde positivamente a mayores niveles de inversión en capital humano e investigación. Por su parte Mueller (2006), en una investigación efectuada en 74 zonas de Alemania, comprobó que el capital emprendedor es un buen conductor del conocimiento entre las universidades y el sector industrial, dados los estrechos vínculos existentes entre la difusión del conocimiento y la capacidad de absorción de la industria.

En cuanto al segundo canal (incremento de la productividad), Holtz-Eakin y Kao (2003) constataron la relación existente entre actividad emprendedora y crecimiento económico en Estados Unidos (utilizando los estados como unidad de observación espacial), mostrando cómo las variaciones en la tasa de entrada y salida de las empresas se relacionan con cambios positivos en la productividad.

En cuanto al tercer canal (creación de empleo), Fritsch y Mueller (2008) observaron la existencia a corto y largo plazo de un impacto positivo del emprendimiento sobre la creación de empleo a causa de mejoras en la oferta de la economía regional. Para las zonas con aglomeraciones habría además un mayor impacto en su fase inicial, fuertes efectos a mediano plazo (negativos), y mayores ventajas a largo plazo, siendo determinantes los niveles de producción de las regiones en el efecto del emprendimiento sobre el empleo. Career y Thurik (2008), en un estudio realizado en los países de la Organización para la Cooperación y el Desarrollo Económicos (OCDE), concluyeron sin embargo que la existencia de las etapas de afectaciones del emprendimiento tienen importantes impactos sobre la productividad y el PIB, mas no sobre los niveles generales de empleo. En este ámbito, Mueller, Van Stel y Storey (2008) determinaron la incidencia del emprendimiento en la generación de empleos en Gran Bretaña, explicando la dependencia de las condiciones de las regiones -educación, acceso a crédito y posesión de activos- para la existencia de impactos positivos del emprendimiento.

Teniendo en cuenta los tipos de empresas, Acs y Mueller (2008) analizaron los efectos de la nueva dinámica de los negocios en el nivel de empleo regional, demostrando empíricamente la importancia de la existencia de condiciones heterogéneas en el mercado laboral-empresas ratón, gacelas 
y elefantes- en el tiempo. Adicionalmente, probaron que las características de cada región afectan el éxito de uno u otro tipo de emprendimiento. Por su parte, Salas y Sánchez (2006), al intentar establecer una relación entre emprendimiento y desarrollo económico mediada por el incremento de la productividad, identificaron la administración del grupo emprendedor de alta calidad -innovación, resultante de las condiciones preexistentes positivas basada en el acceso a educación- como fuente de mayores niveles de producción. En el caso particular de España, Massón (2007) confirmó la importancia del capital emprendedor en la economía, básicamente cuando es generado por empresas de menor dimensión, señalando que a mayor nivel de capital emprendedor, mayor será el número de empresas nuevas, siendo las de menor tamaño las que más contribuyen en las funciones de producción y de conocimiento: el capital emprendedor que genera pequeñas empresas -de uno a cinco empleados- ejerce un impacto favorable en la productividad laboral, en vista de que utilizan sus recursos y capacidades de manera óptima, desarrollando ventajas competitivas que les permiten supervivir en el sistema.

Wong, Ho y Autio (2005) definieron que el emprendimiento de alto potencial tiene incidencia en el crecimiento económico. El emprendimiento por necesidad $^{2}$ demostró no tener ningún impacto en el crecimiento económico, a diferencia del emprendimiento por oportunidad, ${ }^{3}$ lo cual puede ser explicado por la existencia de economías poco desarrolladas en la muestra. En esta línea de investigación, Van Stel, Carree y Thurik (2007) intentaron evaluar los efectos de las diversas políticas de emprendimiento en los niveles de emprendedores nacientes y nuevos negocios, concluyendo que era importante revisar las conexiones entre las condiciones para el emprendimiento -carga administrativa, tiempo y costos- y la coherencia de determinar las políticas de acuerdo al tipo de emprendedores, sean por oportunidad o necesidad.

Para terminar con el panorama de la literatura internacional, también hay que tomar en consideración el nivel de desarrollo en la relación entre

2. El emprendimiento por necesidad es aquel en donde las personas llevan adelante un negocio que va a generar ingresos que les permitan cubrir sus necesidades básicas en el corto plazo, generalmente desarrollados en el sector informal de la economía.

3. El emprendimiento por oportunidad es aquel en donde las personas empiezan su negocio siguiendo una oportunidad en el mercado, para mejorar sus condiciones laborales y de ingresos, y generalmente tienen perspectivas de desarrollo futuro. 
emprendimiento y crecimiento económico. Wennekers y otros (2005) observaron la existencia de una relación en forma de "U" entre el capital bruto invertido en emprendimiento y el desarrollo y bienestar económico. Así, la innovación inicialmente desincentiva los nuevos negocios, sin embargo, después de encontrar mayor estabilidad impulsa los emprendimientos. Un mayor número de propietarios de negocios estimulan la creación de emprendimientos nacientes, en tanto que una seguridad social generosa mostró tener impactos negativos sobre el emprendimiento. En conclusión, la dinámica del emprendimiento tiene un impacto diferente en el crecimiento económico, de acuerdo a la etapa de desarrollo de un país, por lo que debería ser considerada para la formulación de una política económica tomando en cuenta la tasa natural del emprendimiento que es más alta en países de ingreso bajo que en países de ingreso medio y alto. Por el contrario, Van Stel, Carree y Thurik (2005) sugieren que no existe un cambio significativo en el nivel de influencia del emprendimiento sobre el crecimiento de acuerdo al nivel de desarrollo, si bien es algo más intensa en el caso de las economías más avanzadas. Mientras que Stam y otros (2006) evaluaron la incidencia de los emprendedores en los niveles de crecimiento económico en diversos tipos de economías, señalando que el impacto de estos es considerable en el crecimiento económico, especialmente en economías en transición.

En América Latina existen muy pocos estudios que traten las relaciones entre el emprendimiento y el crecimiento de la economía en general y de la productividad en particular. Una reciente publicación del Banco Mundial (BM) a cargo de Lederman y otros (2014) ha puesto de manifiesto que en la región se crean muchas pequeñas empresas, pero con muy poca innovación. Entendiendo al emprendimiento según los preceptos de Schumpeter, como la entrada de empresas en mercados nuevos o ya establecidos, la introducción de productos nuevos en el mercado y los avances organizativos que permiten a las empresas mejorar la calidad o el precio de los productos o adoptar maneras de producir más eficientes, los autores ponderan en su investigación que la medición del emprendimiento no es una tarea sencilla, ya que está relacionada con los rasgos y aptitudes individuales de una pequeña élite de empresarios.

Kantis y Federico (2012) y Kantis, Federico y Menéndez (2012) en sus estudios sobre los emprendimientos dinámicos en América del Sur, efectua- 
dos para definir la existencia de factores sistémicos que inciden en el surgimiento y desarrollo de este tipo de emprendimientos, estimaron que existe una influencia directa de la cultura y el sistema educativo en la formación de capital humano emprendedor, que se requiere también una estructura productiva dinámica y diversificada, pero que se aprecia una escasa incidencia de las plataformas de ciencia y tecnología y del financiamiento en la creación de negocios.

El desarrollo del emprendimiento en la región también ha sido abordado por Acs y Correa (2014), quienes concluyeron que el financiamiento en condiciones adecuadas es uno de los principales limitantes del emprendimiento en países en desarrollo, en donde la educación y la asistencia técnica deberían ser pilares básicos en el fomento de emprendedores dinámicos, siempre y cuando la estrategia de innovación llegue a todos los sectores económicos. De manera particular, un estudio efectuado en Chile por Poblete y Amorós (2013) para evaluar el impacto que tiene la educación universitaria en el desarrollo de emprendedores, señaló que existe una reducida interacción entre emprendedores y universidades, por lo que no se aprecia un real impacto en la actividad emprendedora, razón por la cual, la educación universitaria enfocada en el emprendimiento no aumentaría la probabilidad de tener intenciones de emprender.

En lo que respecta a Ecuador, tampoco existen estudios que demuestren la relación entre capital emprendedor y crecimiento económico de manera inequívoca. A pesar de ello, una investigación reciente efectuada por Lara y Flor (2013) sobre el capital emprendedor y su incidencia en el desarrollo socioeconómico de la región central del Ecuador, permite deducir que las provincias más emprendedoras y con mayor crecimiento son aquellas en las cuales existe una fuerte presencia de políticas de apoyo, así como las que cuentan con mayores niveles de infraestructura, educación y salud.

En resumen, a la vista del estado de la literatura se puede concluir que la relación entre capital emprendedor y crecimiento económico es positiva pero no incondicional, ya que depende de otros determinantes como innovación, financiamiento y educación, que influyen directamente en el emprendimiento, así como de las políticas públicas y el entorno institucional -clima de negocios- que lo hacen indirectamente. 


\section{Evaluación de los indicadores del emprendimiento}

Nightingale y Coad (2014) señalan que el impacto de la iniciativa empresarial en la economía y el rendimiento relativo de las empresas emprendedoras es una cuestión empírica sujeta a numerosos problemas metodológicos, que remiten a la vieja diferencia entre asociación y relación de causalidad. En lo que sigue se trata de afrontar este desafío mediante un análisis tipológico de los diez principales índices y dashboards que permiten aproximar el fenómeno del emprendimiento de manera directa o indirecta, teniendo en cuenta su nivel de cobertura, las dimensiones consideradas, y el método de cálculo o presentación de datos para medir el emprendimiento. Estos indicadores han sido elaborados por instituciones internacionales como el Banco Mundial, la OCDE, el Foro Económico Mundial (FEM), el Instituto Global de Emprendimiento y Desarrollo (IGEP), el EIM-Business \& Policy Research de Holanda y universidades como el Babson College, Cornell y el Prodem de la Universidad General Sarmiento de Argentina. Para realizar una aproximación a su tipología se sistematizaron tres grupos de indicadores, tomando en cuenta el nivel de precisión en la medición, de mayor a menor, en razón de que existen indicadores que reflejan de manera directa o indirecta al emprendimiento, presentados como índices compuestos o tableros de datos.

El Grupo I está integrado por tres índices compuestos que aproximan directamente el emprendimiento pero con desigual cobertura temporal y geográfica: Global Entrepreneurship Monitor (GEM, realizado desde 1998; actualmente para 137 países, 19 de América Latina y el Caribe-ALC), Global Entrepreneurship and Development Index (GEDI, realizado desde 2008; actualmente para 130 países, 25 de ALC), e Índice de Condiciones Sistémicas para el Emprendimiento Dinámico (ICSED, realizado desde 2014; actualmente para 54 países, 15 de ALC).

El Grupo II está integrado por tres dashboards que también aproximan el emprendimiento directamente, con una cobertura temporal asimismo desigual, pero geográficamente mucho más limitada que los indicadores del Grupo I, por cuanto no incluyen países de América Latina y el Caribe: Comparative Entrepreneurship Data for International Analysis (COMPENDIA, realizado desde 1970; actualmente para 30 países), International Benchmark of Entrepreneurs (IBE, realizado desde 1995; actualmente para 17 países) 
y Eurostat Entrepreneurship Indicators Programme (EIP, realizado desde 2011; actualmente para 34 países).

Finalmente, el Grupo III está integrado por índices compuestos y un dashboard que aproximan el emprendimiento indirectamente, con una cobertura temporal dilatada y geográficamente amplia: Índice Global de Competitividad (IGC, realizado desde 1979; actualmente para 144 países, 19 países de ALC), Doing Business (DB, realizado desde 2004; actualmente para 189 países, 32 de ALC) y Global Innovation Index (GII, realizado desde 2007; actualmente para 141 países, 22 de ALC) como índices compuestos; y el World Bank Group Entrepreneurship Snapshots (WBGES, realizado desde 2004; actualmente 125 países, 22 de ALC), dashboard que, pese a su denominación, es una aproximación muy indirecta al emprendimiento.

Es importante señalar que el objetivo de la creación de estos indicadores es determinar la situación del emprendimiento y ayudar a los gobiernos, o proveerlos, de una herramienta de análisis que les permita desarrollar políticas públicas de fomento productivo. Las bases de datos e indicadores compuestos generados buscan también mantener un alto nivel de comparabilidad entre los países, aunque no manejen unidades de medición común ni definiciones consensuadas de emprendimiento o fuentes de información estandarizadas para todos los países.

\section{Fuentes de información}

Los diez indicadores utilizan fuentes de información muy diversas, que se pueden clasificar en objetivas y subjetivas. Las primeras pueden ser cuantitativas o cualitativas. Las cuantitativas están tomadas de las diferentes mediciones presentadas de manera oficial en los países analizados; por otra parte, existen otro tipo de datos que son numéricos y con determinaciones fijas, como es el caso del cálculo de costos para procesos específicos, lo cual se basará en tarifarios locales, que deberán pasar por procesos de cuantificación claros para que sean comparables en los diferentes países y estén en función de la realidad. Las fuentes objetivas cualitativas, por su parte, provienen de aquellos datos que pueden ser contrastados contra documentos como leyes o regulaciones específicamente. Finalmente, las fuentes subjetivas proceden 
de los resultados de encuestas de opinión ejecutiva o efectuada a expertos, generalmente del sector privado.

Dada la proliferación de índices compuestos y bases de datos, muchos de estos a su vez utilizan datos de otros indicadores. El GEDI, por ejemplo, usa el IGC y el GEM, además del Índice de Percepción de Corrupción de Transparencia Internacional, el de Inserción a la Educación Superior (GERD) de la UNESCO, el de Uso de Internet de la Asociación Internacional de Telecomunicaciones, el Índice de Urbanización de Naciones Unidas, el de Globalización Económica (KOF) del Instituto Suizo de Economía, o el de Riesgo de Clima de Negocios (COFACE) de la Conferencia de Riesgo País. Por su parte, el ICSED toma en cuenta las mejores prácticas de construcción de índices recomendadas por la OCDE y sus fuentes de información se eligen por provenir de bases de datos confiables y legitimadas internacionalmente, entre otras las del GEM, IGC, UNESCO y la Encuesta Mundial de Valores.

En los dashboards del Grupo II (COMPENDIA, IBE y EIP) se compiló indicadores estandarizados entre los diversos integrantes de la OCDE, que permitieran mantener en una sola base de datos la información internacional acerca del emprendimiento. Estos datos provienen directamente de fuentes oficiales de los países (por ejemplo, de los registros nacionales de constitución de empresas), pero adicionalmente usan otras bases de datos reconocidas como el Eurostat, Compustat, o Amadeus, y también recurren a instituciones reconocidas por su experticia en temas de emprendimiento para legitimar los resultados alcanzados, como la Organización Mundial de Comercio (OMC). De igual manera, los diferentes indicadores que se calculan con estos datos sirven para sugerir estrategias de fomento productivo y crecimiento en cada economía. Por el momento, este tipo de indicadores son difíciles de construir para países en desarrollo debido a las deficiencias de los sistemas estadísticos nacionales.

En el Grupo III está el caso específico del WBGES, que sí es un dashboard con cobertura para países en desarrollo, la principal fuente de información es provista por los institutos nacionales de estadísticas. Para obtener la información, la WBGES prepara una encuesta que es remitida a los diferentes países y posteriormente validada o auditada por el Banco Mundial para garantizar su fiabilidad. En los casos de ausencia de información se utilizan otras fuentes nacionales alternativas o indicadores internacionales reconocidos. 
Dentro también del Grupo III, el GII se basa en datos de la UNESCO, OCDE, FMI e ISO y cuenta con una mesa de asesoría, que está conformada por un grupo de líderes internacionales y expertos que revisan la metodología y siguen de cerca todas las acciones realizadas para el cálculo del índice, que es sometido a una auditoría estadística independiente realizada por el Joint Research Center de la Unión Europea.

\section{Dimensiones, métodos de cálculo y presentación}

Dentro del Grupo I, el GEM mide los niveles de emprendimiento mediante la tasa de emprendimiento temprano (TEA), que se estima considerando la incidencia de personas que constituyeron negocios nuevos, es decir, de más de tres meses de duración, pero menos de tres años y medio; y se adicionan las personas que establecieron o buscan en el corto plazo establecer negocios nacientes, menores a tres meses $-\mathrm{y}$ que en muchas ocasiones solo se evalúan las intenciones de emprender-, comparando esta suma con la población en capacidad de instaurar un negocio. La TEA ha sido hasta ahora el indicador de mayor comparabilidad entre las distintas economías para evidenciar el grado de actividad emprendedora, y el GEM lo completa con otros índices como la tasa de emprendimientos nacientes y la tasa de cierre de negocios.

Es importante subrayar que el GEM se enfoca directamente en las personas y sus expectativas, considerando de manera complementaria los factores externos que influyen en el desarrollo empresarial. En tal sentido analiza requerimientos básicos, potenciadores de eficiencia y aspectos de innovación. Para este último indicador, cuanto más avanzado sea el grado de desarrollo de la economía analizada mayor será el aporte del emprendimiento en la misma y evalúa su percepción en función de nueve variables específicas: políticas gubernamentales, programas gubernamentales, educación y capacitación, financiamiento, infraestructura comercial, regulaciones culturales o sociales, infraestructura física, apertura del mercado interno, y transferencia de investigación y desarrollo. El GEM recopila sus datos a partir de la encuesta de población adulta (APS), en la que los equipos de investigación acreditados de cada país deben realizar la indagación a una población de al menos 2.000 personas por año. 
El objetivo de la APS es identificar la capacidad emprendedora, la motivación, la percepción y las aspiraciones de las personas, para extrapolar sus resultados a nivel nacional. Esta encuesta utiliza preguntas cerradas y es aplicada a personas entre 18 y 64 años, a pesar de que es aceptado encuestar en un rango etario mayor, considerando la heterogeneidad de las personas participantes que se involucran en responder las preguntas. En la APS se evalúan diferentes tipos de emprendedores, puesto que mientras algunos individuos pueden estar aspirando a iniciar un negocio, otros pueden mantener un negocio en marcha y tener diversos criterios frente al emprendimiento, quienes deben ser aglutinados a efectos de tener un resultado único nacional.

Por su parte, el GEDI reconoce que el emprendimiento puede tener un impacto tanto a nivel económico como social en las personas, motivo por el cual el indicador capta información de un contexto individual -actitudes, capacidades y aspiraciones-y también de la interacción de estos tres factores con el ámbito institucional. Se trata, por tanto, de un índice compuesto de carácter ecosistémico. El indicador compuesto sintetiza catorce variables: percepción de oportunidades, habilidades para iniciar empresas, aceptación del riesgo, networking, apoyo cultural, oportunidades de desarrollo de startups, absorción tecnológica, capital humano, competencia, innovación de productos, innovación de procesos, alto crecimiento, internacionalización y capital de riesgo.

En el GEDI todas las variables dependen de otras, por lo cual utiliza un algoritmo para penalizar la calificación de algunos componentes si otros relacionados tienen un rendimiento negativo o bajo. Previo a esto, el GEDI parte de una matriz de correlación de variables, que determina los pesos con los que se relacionan unas con otras al examinar esas interrelaciones, trata de identificar cuellos de botella que serán a la vez hojas de ruta para los países en pos de mejorar su productividad o competitividad. Para llegar al indicador compuesto, en primer lugar se realiza el levantamiento de datos conforme a las fuentes de datos previamente mencionadas, después cada variable es normalizada en un rango entre 0 y 1 . Posteriormente, se ajustan los resultados de cada variable para que sean comparables, por lo cual el ajuste considera la comparación del resultado obtenido frente al 95\% de datos obtenidos de todos los países de acuerdo a una distribución estadística hecha para cada va- 
riable, es decir, se ajustan los datos considerando el promedio de resultados observados.

Para cerrar el Grupo I, el ICSED, a más del análisis multidimensional propuesto por índices ampliamente reconocidos como el IGC o el DB y del análisis ecosistémico propuesto por el GEDI, incorpora como concepto céntrico el emprendimiento dinámico o empresas gacelas. Este tipo de negocio se caracteriza por su potencialidad productiva, intensidad tecnológica o de innovación, por su mayor capacidad de generación de empleo y su dinamismo a la hora de adaptarse al mercado. El ISCED sintetiza 41 variables agrupadas en diez dimensiones de gestión directa que requieren la atención de la política pública, y que son sociales, culturales y económicas: capital humano emprendedor, cultura, condiciones sociales de la familia, sistema educativo, condiciones de la demanda, estructura empresarial, plataforma de ciencia y tecnología para la innovación, financiamiento, capital social (redes) y políticas y regulaciones.

Para calcular el ICSED se realiza un promedio móvil de sus últimos dos valores disponibles, dentro de un período que abarca los tres últimos años. La razón de este promedio móvil es eliminar una probable aleatoriedad de cada variable analizada, especialmente de aquellas que tienen alta subjetividad en su medición, como es el caso de las encuestas que se realizan a informantes claves en el GEM o en el IGC. Análogamente al GEDI, en el caso en que no encuentren valores para determinadas variables, el ICSED toma en cuenta a expertos en el tema, y adicionalmente, en otros casos, utiliza los valores de economías comparables, siempre y cuando dichas semejanzas se presenten también en las otras variables de la misma dimensión. Posterior a la cuantificación de las variables se realiza una normalización siguiendo el método Máx-Mín, que consiste en medir la distancia relativa de cada variable de cada país frente a la variable del país con peores condiciones y reestimar estas distancias en un rango entre 0 y 1.

En el Grupo II, el COMPENDIA, IBE e EIP toman en cuenta aspectos relevantes para el emprendimiento surgidos de una serie de modelos económicos y analíticos realizados en el entorno de la OCDE, así como los determinantes de la estructura del emprendimiento de sus diversos países integrantes, basándose en el análisis de los propietarios de negocios y su relación con los aspectos laborales, con información acerca de la creación y quiebras de em- 
presas por tamaño y sector económico. Los indicadores centran la atención en la existencia y representatividad del sector productivo en cada país, dentro de este analizan la participación que tienen las empresas de rápido crecimiento o emprendimientos dinámicos. El IBE inicialmente evalúa el comportamiento empresarial de forma anual en función del número de empresas existentes en un mercado, cantidad que varía conforme las entradas, salidas o quiebras que se presentan, y estos datos se complementan con información de empleo generado. Los indicadores de empresas de rápido crecimiento, por su parte, son categorizados en términos del grado de desarrollo que tiene una empresa -dinámico, no dinámico o en crecimiento-, y se evalúan por el promedio de ventas y de empleados que poseen. Estos indicadores son medidos en períodos trianuales.

Por su parte, con el objetivo de estandarizar la información entre los distintos países, el EIP estableció algunas normas para la presentación de resultados: primero, si el indicador se evalúa considerando el tamaño de empresa, dicho tamaño se diferenciará por el número de personas empleadas; mientras que, para los casos en que se evalúen las características de las empresas -su demografía- se las clasificará en función del número de empleadores que hay en el país. Finalmente, cuando el indicador requiere de un análisis por actividad económica, se evalúan únicamente la manufactura, los servicios y la construcción. Para dar seguimiento a la valoración de estos indicadores y su estandarización, la OCDE creó también un grupo de administración de los indicadores de emprendimiento -EISG, por sus siglas en inglés-, el cual reúne a los expertos en políticas de desarrollo emprendedor y a los responsables de generar los datos o información en cada oficina nacional. Dicho grupo desarrolló inicialmente la metodología de cálculo del EIP.

Finalmente en el Grupo III, el IGC se centra en la medición del elemento multidimensional de la competitividad por países, que sintetiza en un solo valor 144 variables agrupadas en 14 pilares o dimensiones: instituciones, infraestructura, ambiente macroeconómico, salud y educación primaria, educación superior y entrenamiento, eficiencia de los mercados de bienes, eficiencia del mercado laboral, desarrollo del mercado financiero, preparación tecnológica, tamaño del mercado, sofisticación de los negocios y, finalmente, la innovación. El IGC normaliza los resultados de sus encuestas en una escala del 1 al 7 y realiza un promedio respecto al año anterior, categorizando a 
los diferentes países en relación con el nivel de desarrollo productivo de sus economías. El sistema de agregación de variables a subíndices y de subíndices al indicador compuesto es la media aritmética ponderada, no obstante, el peso que se da a cada pilar y subindicador es variable, y depende del tipo de economía analizada; así, por ejemplo, en economías en proceso de desarrollo el peso de requerimientos básicos es del $60 \%$, los potenciadores de eficiencia del 35\% y los factores de innovación del 5\%.

El DB, por su parte, analiza el entorno normativo, la calidad, la transparencia de las regulaciones existentes y el aporte institucional para el desenvolvimiento de este entorno, ya que es un transitar que necesariamente deberá enfrentar toda empresa durante su ciclo de vida, independientemente de su tamaño, y que puede ser determinante a la hora de mejorar su competitividad interna, la productividad de la economía y el potencial para la creación de puestos de trabajo. El DB sintetiza en un indicador compuesto por diez variables que facilitan el clima de negocios: apertura de una empresa, manejo de permisos de construcción, obtención de electricidad, registro de propiedades, obtención de crédito, protección de inversores, pago de impuestos, comercio transfronterizo, cumplimiento de contratos y resolución de la insolvencia. Para el cálculo del DB se utiliza una metodología que analiza la normativa aplicable a una empresa tipo que opera en la ciudad más relevante de una economía determinada, es decir, se realiza un estudio de caso estándar por país, proceso durante el cual se evalúan todas las regulaciones a las que ha sido afectada la empresa, luego aplica las encuestas a especialistas y posteriormente se validan los datos con los gobiernos de los diferentes países, conjuntamente con el personal regional del BM.

Y el grupo GII se centra en medir la innovación de manera también multidimensional a partir de un índice compuesto que sintetiza 81 variables en siete dimensiones, cinco referidas a inputs: instituciones, capital humano e investigación, infraestructura, complejidad de mercado y complejidad de empresas y dos a outputs: conocimiento y tecnología, y creatividad. Dado que la concepción del GII mantiene el enfoque del proceso de innovación, diferenciando por entradas y salidas, de igual manera se procede a su cálculo que se compone de dos subíndices: insumos de la innovación (entradas) y productos de la innovación (salidas). Para el cálculo se utiliza como método de agregación el promedio ponderado de las dimensiones posterior a una 
estandarización de los mismos, que deja los resultados en un rango entre 0 y 100, utilizando el método Máx-Mín. Cada subpilar se compone de tres a cinco indicadores, entre los cuales se calcula el promedio ponderado, y de la misma manera cada pilar es valorado por el promedio ponderado de sus diferentes subpilares. Posteriormente se establecen los resultados de los dos subíndices, que pueden considerarse unas primeras aproximaciones reales a la situación del emprendimiento en un país y están valorados por el promedio simple de los pilares que conforman cada uno de los subíndices. Finalmente se calculan dos índices generales: el ratio de eficiencia de la innovación, evaluado por la suma de las puntuaciones de cada subíndice; y el índice global de innovación, calculado mediante un promedio simple de los dos subíndices mencionados.

Por último, en el Grupo III, el WBGES es una base de datos que contiene indicadores relevantes sobre la entrada de nuevas empresas en una economía y sobre la densidad de estos emprendimientos. Pretende desarrollar indicadores específicos que permitan evidenciar la situación de la creación de empresas en un país, considerando que existe evidencia empírica de que un sector privado fuerte tiene un impacto positivo sobre el desarrollo económico, y para esto, el progreso de este sector dependerá del comportamiento de las normas, políticas e instituciones existentes. Analiza únicamente las empresas que han sido formalmente creadas, es decir, cumpliendo las normas y un reconocimiento jurídico sin importar su tamaño. Esta decisión implica que se deje de lado a todo el sector informal o subempleado. Para el cálculo del WBGES, en un primer momento se reúnen los datos de la totalidad de empresas formales que han sido creadas en una economía, considerando únicamente a la modalidad de responsabilidad limitada, al igual que en el DB. Adicionalmente, se analiza la densidad de empresas nuevas, definidas como el número de sociedades de responsabilidad limitada de nueva constitución, por cada 1.000 personas en edad de trabajar, es decir, entre 15 y 64 años.

Además de los problemas clásicos de agregación de índices compuestos e interpretación de bases de datos, conviene señalar para concluir este apartado que existen dos limitantes adicionales al uso de indicadores empleados para aproximarse al emprendimiento como determinante del crecimiento económico: el primer limitante se asocia al sesgo que provoca la omisión de variables cualitativas de difícil cuantificación o a la carencia de variables 
cuantitativas en algunos países o períodos específicos; el segundo es el efecto que pueden tener las circunstancias coyunturales que atraviesan algunos países en vías de desarrollo, que son producto del entorno y alteran el impacto de las variables explicativas que se utilizan en las distintas metodologías aplicadas para el cálculo de los indicadores y que están sesgadas en el plano normativo a los entornos institucionales de los países desarrollados (Chang 2011).

\section{Balance de ventajas y desventajas de los principales indicadores}

La mayoría de los indicadores evaluados en el presente documento pueden usarse como base de análisis, por su carácter multidimensional (todos los del Grupo I, y los índices compuestos del Grupo III, especialmente). Utilizando sus diversos componentes se pueden realizar comparaciones entre las realidades de los países, pese a que algunas de esas dimensiones puedan ser ejes transversales al emprendimiento, como es el caso de la innovación, la sofisticación de los negocios, el desarrollo de los mercados laborales y financieros, la calidad de las instituciones o el entorno macroeconómico.

Hay seis indicadores que acumulan más de una década de generación de datos (GEM, COMPENDIA, IBE, IGC, DB y WBGES), lo que permite elaborar análisis de tendencias reales de las distintas variables de fomento productivo y eventualmente identificar causas de crisis económicas. Al no depender exclusivamente de las estadísticas oficiales de los países y sus constantes variaciones metodológicas de cálculo, arrojan además un alto grado de comparabilidad intertemporal.

Dado que todos, excepto el GEM, se enfocan en las empresas y entornos socioeconómicos, no en los individuos, se obtiene un panorama más real y completo del emprendimiento en cada país. Esto remite al carácter ecosistémico de la medición de indicadores, como ocurre con el GEDI y el ISCED: si un ecosistema es un conjunto de elementos interrelacionados que soportan el desarrollo emprendedor, se trata entonces de tener un panorama más completo de análisis y evidenciar otro tipo de factores que pueden representar rutas de acción para las economías. Un aspecto fundamental de manejar un eco- 
sistema es la interacción que existe entre las diferentes variables y el efecto que pueden tener unas sobre otras. Este tipo de análisis de sensibilidad tiene una importancia especial puesto que sirve como un mecanismo para simular diferentes escenarios de política pública. En este sentido, un tema importante para mantener un enfoque sistémico es considerar un entorno de fomento del emprendimiento que se encuentra constituido por variables de carácter estructural, es decir, vitales para los países, como el sistema educativo o las condiciones sociales que, independientemente del grado de desarrollo de la economía, merecen una gestión diaria.

La densidad empresarial, es decir, el número real de empresas que están interactuando en cada economía, es otro elemento a tener en cuenta de indicadores como el WBGES. Este, además de la quiebra y las salidas de empresas, da una medida de que el emprendimiento no incide únicamente en la creación de empresas, sino que puede tener importantes limitaciones a lo largo de la implementación de una compañía que pueden ser determinantes para salir del mercado. Dentro de esto, el análisis de la innovación permite tener una visión más amplia y no una aplicación estrictamente empresarial, por lo cual, es importante mostrar otro tipo de factores que no son tradicionalmente considerados dentro de los índices de emprendimiento clásicos, como por ejemplo, analizar la creatividad de los productos o servicios que se están desarrollando dentro de una economía.

Por último, un elemento digno de resaltar es que todos los indicadores excluyen la información de los países que mantienen centros offshore, debido a que en muchas ocasiones los datos provenientes de estos centros no se originan en emprendimientos como tal, más bien son empresas creadas únicamente con fines de atracción de inversiones externas, con objetivos ilegales como la evasión fiscal, o representan negocios ficticios puesto que no están realmente activos dentro de estos países.

En cuanto a las principales desventajas de los indicadores evaluados, la primera hace referencia a la falta de estandarización de clasificaciones y conceptos, desde la relativa al tamaño de las empresas a términos como autoempleo e innovación, con el riesgo de que se esté realizando una comparación entre variables totalmente disímiles. También se pueden criticar las razones de inclusión o exclusión de ciertas variables, asociadas a determinados conceptos -como la competitividad en el ICG-, que en ocasiones se pueden consi- 
derar colaterales o irrelevantes y esto puede llegar a diluir el verdadero aporte de otros factores que sí tienen una incidencia directa en el emprendimiento.

Otra desventaja se deriva de la medición de las percepciones a partir de encuestas de opinión ejecutiva a personas que a menudo comparten un consenso normativo, por lo tanto, el valor generado se convierte en un indicador de resultados predecibles. En este mismo sentido, otra desventaja de algunos indicadores es su carácter tautológico, el cual se evidencia en la metodología utilizada para clasificar a los diferentes países -en función del PIB per cápita-, lo que a su vez determina los pesos o ponderaciones que tendrá cada dimensión analizada en su agregación a los resultados definitivos por país, como ocurre en el ICG. Esta mencionada metodología y forma de determinación de ponderaciones hará que los resultados estén influenciados por realidades previamente direccionadas, o, en otras palabras, son resultados sesgados con anterioridad. La debilidad metodológica también atañe a variables cualitativas que se basan en percepciones de expertos y no en la utilización de modelos econométricos para comprobar los resultados encontrados. Las metodologías de medición pueden evaluar muy pocas variables, sin tener en cuenta factores que determinan el clima de negocios, como la situación macroeconómica, la calidad institucional o la logística y el transporte. Incluso dentro de las mismas regulaciones no se analizan aspectos como la corrupción o la seguridad jurídica, que son elementos claves de desenvolvimiento del tema normativo.

En el caso concreto del DB, no se puede partir únicamente del análisis de un caso de estudio estandarizado en la principal localidad de negocio de los países seleccionados, ya que se invisibilizan las diferencias y brechas entre áreas rurales y urbanas típicas de los países en desarrollo, dando lugar a una información altamente sesgada. Siguiendo con los aspectos metodológicos, es importante reconocer que si bien la medición de las intenciones de emprender que hace el GEM puede presentar un panorama más completo, el análisis de este componente debe ser utilizado con cautela: se trata de aspiraciones a emprender, que no tienen un impacto real en el crecimiento económico, y que pueden estar altamente influenciadas por circunstancias económicas específicas, como por ejemplo una recesión local, que incrementará notablemente los emprendimientos por necesidad. 
Algunos indicadores por su carácter reciente (menos de diez años, como es el caso de GEDI, ICSED, EIP y GII) todavía no cuentan con un número de observaciones anuales relevantes para llevar a cabo un análisis econométrico significativo, por lo cual, sus resultados en ocasiones pueden estar influenciados por los ciclos económicos propios de los análisis circunstanciales o que solo consideran períodos actuales. También se ha podido observar que varios indicadores no recopilan datos de manera individual únicamente, sino que la complementan con datos provenientes de expertos, o peor aún, originados en otros indicadores con otras metodologías y limitaciones. En este caso, se replica la desventaja derivada de la aplicación de encuestas a expertos con similares prácticas profesionales, lo que hace que los resultados sean previsibles.

Una desventaja adicional también relevante es la falta de disponibilidad de información de las variables consideradas institucionales. Si bien se considera que incrementar el aporte de la institucionalidad es interesante para definir un indicador, la medición de este factor tiene amplias salvedades, ya sea por la ausencia de datos comprobables, por la dispersión de datos, por la atemporalidad de los datos encontrados o por los juicios normativos ocultos en la generación de los mismos mediante encuestas a expertos.

Otra desventaja importante es que los ecosistemas de emprendimiento presentes en varios indicadores no han llegado a ser comprendidos en su totalidad, es más, están en nacimiento, y todavía no se sabe a ciencia cierta qué factores tienen incidencia directa en el desarrollo emprendedor, existiendo alta complejidad para su cuantificación, y peor si ello se hace con información rígida, es decir, con datos de base tomados de otros indicadores. Con este criterio, los elementos que sirven en una economía pueden tener resultados completamente distintos en otra, pudiendo a veces obtener respuestas incongruentes en ciertos países, para lo cual es necesaria la inclusión de otro tipo de datos de carácter cualitativo.

Una crítica particularmente relevante de los indicadores analizados para su aplicación en los países en desarrollo es que los resultados que muestran se limitan generalmente al sector formal de la economía, es decir, a las empresas legalmente constituidas. Sin embargo, los países en desarrollo se caracterizan por tener un sector informal significativo en términos de PIB y sobre todo de empleo, y que tiene un aporte al dinamismo económico. Es comprensible 
que el sector informal, por sus características, tenga mayores dificultades de cuantificación del número y calidad de las empresas, sin embargo, su representatividad no puede descartarse. De igual manera, el hecho de considerar solo a las empresas nuevas y formalmente creadas no implica que en realidad estos negocios han sido emprendidos, ya que algunos pueden estar recién formalizados aunque hayan venido operando informalmente durante años. Adicionalmente, estas compañías pueden desenvolverse en sectores tradicionales y pueden existir empresas liquidadas constantemente, pero como únicamente se evalúan las entradas no se sabe en realidad qué tan competitivas puede llegar a ser en una economía solo basándose en el stock de negocios creados.

En este sentido, hay que considerar que por lo general las bases de datos expresan información únicamente de las empresas de responsabilidad limitada que han sido constituidas en un país, dejando automáticamente excluidas a las sociedades anónimas u otro tipo de modalidad de constitución de empresas privadas que exista en los diferentes países. Es importante mencionar que en los países en desarrollo, especialmente de Latinoamérica, la sociedad anónima o la empresa unipersonal son esquemas utilizados con recurrencia al emprender un negocio y también se podría dejar de lado a empresas públicas o mixtas que en ciertos países son empresas que realmente dinamizan el sector productivo. En relación a estas cuestiones de registro, es importante señalar que existe una diversidad de concepciones en las estadísticas nacionales de cada país sobre la creación de empresas, quiebras y salidas del mercado, por lo que es necesario que los indicadores tengan un factor de corrección de los datos para que puedan ser comparables y no se incurra en importantes sesgos u omisiones. Además, en la información presentada por algunas bases de datos no se considera a las empresas que actúan en el sector agrícola, que puede llegar a ser una actividad económica de amplia relevancia si se utiliza este tipo de metodologías en los países en desarrollo.

Por último, una desventaja de la mayoría de indicadores es que tienen un claro enfoque neoliberal, y evalúan específicamente al sector privado, motivo por el cual se ocultan muchas ineficiencias de Estado, que son recurrentes en muchos países, y que también limitan el clima de negocios, mientras dejan de lado a otros sectores, como el de economía popular y solidaria con potencial innovador y de crecimiento económico. 


\section{Conclusiones}

La evaluación de los diez indicadores que miden la relación entre capital emprendedor y crecimiento económico en el mundo ha demostrado que algunos de ellos no son aplicables en América Latina, ya que son únicamente de uso en los países de la OCDE. Estos indicadores se construyeron a partir de diferentes fuentes de información, desde bases de datos de los propios países, bases de datos de organismos internacionales, hasta encuestas de opinión formuladas a expertos. Algunos de ellos toman en cuenta a todos los sectores productivos, otros solamente al sector privado de las economías; también existen aquellos que únicamente analizan a emprendimientos dinámicos y no a emprendimientos de subsistencia, otros se centran en el sector formal de la economía y no analizan datos del sector informal o autoempleo. De la misma manera, existen diferencias en cuanto a la determinación del tamaño de las empresas y a la conceptualización de las variables analizadas. Lo que se puede apreciar de forma conjunta es que todos sugieren la existencia de impactos positivos del emprendimiento en el crecimiento económico de los países.

La presente investigación también logró determinar un grupo de problemas relativos a la medición del capital emprendedor como aporte al crecimiento económico. Entre los principales se pueden mencionar los siguientes: varias metodologías y conceptos con distintos enfoques teóricos, muchas variables a considerar y muestras no representativas, dificultad para medir aporte de variables cualitativas, no disponibilidad de información uniforme, discrecionalidad e intereses institucionales y el tratamiento de la medición como un asunto estadístico y pocos investigadores con conocimiento en econometría.

De la misma manera, se puede expresar que muchos de los índices del Grupo I y III, a pesar de tener cobertura sobre países en desarrollo, están limitados en su aplicación a las realidades de estas economías, que a menudo se encuentran desabastecidas de información y se enfrentan a sesgos en las metodologías, incluso a nivel de la estandarización de resultados -el tamaño de una empresa pequeña de un país desarrollado puede ser considerado como mediano en una economía en desarrollo-. En consecuencia, las recomendaciones de política pública derivadas de estos indicadores plantean problemas 
en su traslado a los países en desarrollo, que deberían examinar con mucha más cautela las estrategias deducidas de los análisis de dichos indicadores y plantearse la construcción de mediciones mejor adaptadas a esas realidades.

A partir de las ventajas y desventajas reseñadas, se puede aventurar que un buen indicador de emprendimiento debería ser multidimensional, y estar planteado con una metodología de medición más integral, es decir, que ofrezca una amplia cobertura temporal y geográfica, y que busque analizar todo tipo de emprendimientos, no solo los dinámicos, en contraste con las encuestas tradicionales que definen un solo tipo de empresa, un tipo específico de persona, o que se aplican en regiones y/o lugares específicos.

Por el contrario, y más allá de esos límites, la información debería fundamentarse en todos los tipos de empresa por tamaño, incluyendo al sector informal, a las empresas estatales y a las que son sin fines de lucro, realidades particularmente relevantes para los países en desarrollo. Asimismo, el indicador debería tener en cuenta los principales limitantes de un país para desarrollar el emprendimiento y hacerlo sostenible. El análisis de todos los aspectos multidimensionales permitirá que el nuevo indicador sea más eficaz a la hora de orientar la formulación de políticas públicas de fomento del emprendimiento.

\section{Referencias}

Acs, Zoltan, y Attila Vargas. 2005. "Entrepreneurship, Agglomeration and Technological Change”. Small Business Economics 24, No. 3: 171-186.

Acs, Zoltan, y otros. 2005. "Growth and Entrepreneurship: An Empirical Assessment", Group Entrepreneurship, Growth and Public Policy, Discussion Paper Series, No. 5409: 1-30.

Acs, Zoltan, y Pamela Mueller. 2008. "Employment Effects of Business Dynamics: Mice, Gazelles and Elephants". Small Business Economics 30, No. 1: 85-100.

Acs, Zoltan, y Paulo Correa. 2014. "Identifying the Obstacles to High Impact Entrepreneurship in Latin America and the Caribbean". The Innovation Policy Platform, No. 5: 1-36.

Audretsch, David, y Erik Monsen. 2008. "Entrepreneurship Capital: A Regional, Organizational, Team and Individual Phenomenon". En Rowena Barret y Susan Mason, edit., International Handbook of Entrepreneurship and HRM: 47-70. Montpellier: Edward Elgar.

Audretsch, David, y Max Keilbach. 2004. "Does Entrepreneurship Capital Matter?". Entrepreneurship Theory and Practice 28, No. 5: 419-429. 


\section{Gary Flor}

---. 2005. "Entrepreneurship Capital-determinants and Impact on Regional Economic Performance". Documento de trabajo, CEPR No. 4905. Consulta: mayo de 2016. «https://papers. ssrn.com/sol3/papers.cfm?abstract_id=734405.

Audretsch, David, y otros. 2006. "Growth and Entrepreneurship: An Empirical Assessment". Centre for Economic Policy Research, No. 5409: 12-23.

Career, Martin, y Roy Thurik. 2008. "The Lag Structure of the Impact of Business Ownership on Economic Performance in OECD Countries". Small Business Economics 30, No. 1: 101-110.

Chang, Haj. 2011. "Institutions and Economic Development”. Journal of Institutional Economics 7, No. 4: 473-498.

Fritsch, Michael, y Pamela Mueller. 2008. "The Effect on New Business Formation on Regional Development Over Time: The case of Germany". Small Business Economics 30, No. 1: 15-29.

Holcombe, Randall. 1998. "Entrepreneurship and Economic Growth". Quarterly Journal of Austrian Economics 1, No. 2: 45-62.

Holtz-Eakin, Douglas, y Chihwa Kao. 2003. "Entrepreneurship and Economic Growth: The Proof is in the Productivity". Center for Policy Research Paper, No. 111: 1-34.

Julién, Pierre. 2005. Emprendimiento regional y economía del conocimiento. Cali: Pontificia Universidad Javeriana.

Kantis, Hugo, Juan Federico y Cecilia Menéndez. 2012. "Políticas de fomento al emprendimiento dinámico en América Latina: tendencias y desafíos". Documento de trabajo, CAF No. 2011/09. Consulta: mayo de 2016. 〈https://www.caf.com/media/4233/politicas-em prendimiento-dinamico-america-latina.pdf〉.

Kantis, Hugo, y Juan Federico. 2012. ¿Emprendimientos dinámicos en América del Sur?: la clave es el (eco) sistema. Buenos Aires: Red Mercosur de Investigaciones Económicas.

Lara, Alberto, y Gary Flor. 2013. El capital emprendedor y su influencia en el desarrollo socioeconómico en la región central del Ecuador. Quito: Editorial Ecuador.

Lederman, Daniel, y otros. 2014: El emprendimiento en América Latina: muchas empresas y poca innovación. Washington D.C.: Banco Mundial.

Marshall, Alfred. 1916. Principles of Economics. Londres: MacMillan.

Massón, José. 2007: "El capital empresarial como determinante de la productividad y el crecimiento en España”. Munich Personal Repec Archive Paper, No. 4073: 1-36.

Mueller, Pamela. 2006. "Entrepreneurship in the Region: Breeding Ground for Nascent Entrepreneurs". Small Business Economics 27, No. 1: 41-58.

Mueller, Pamela, André Van Stel y David Storey. 2008. "The Effects of New Firms Formation on Regional Development Over Time: The case of Great Britain”. Small Business Economics 30, No. 1: 59-71.

Naudé, Wim. 2013. "Entrepreneurship and Economic Development: Theory, Evidence and Policy". Documento de trabajo, IZA, No. 7507: 5-20. Consulta: abril de 2016. «http://ftp. iza.org/dp7507.pdf〉. 
Nightingale, Paul, y Alex Coad. 2014. "Muppets and Gazelles: Political and Methodological Biases in Entrepreneurship Research". Industrial and Corporate Change 23, No. 1: 113143.

Poblete, Carlos, y José Amorós. 2013. "University Support in the Development of Regional Entrepreneurial Activity: An Exploratory Study from Chile". Investigaciones Regionales, No. 26: 159-177.

Roudini, Azizallah. 2012. "Global Economic Crisis and Entrepreneurship Development". International Journal of Fundamental Psychology \& Social Sciences 2, No. 1: 13-18.

Rüdiger, Klaus, Marta Peris-Ortiz y Alicia Blanco Gonzales. 2014. Entrepreneurship, Innovation and Economic Crisis Lessons for Research, Policy and Practice. Gewerbestrasse: Springer International Publishing.

Salas, Vicente, y Javier Sánchez. 2006. "Entrepreneurship, Management Service and Economic Growth". Group Entrepreneurship, Growth and Public Policy 45, No. 17: 1-24.

Stam, Erik, y otros. 2006. "Los emprendedores con potencial de crecimiento y desarrollo económico: Políticas públicas de apoyo a los emprendedores". Ekonomiaz, No. 62: 124-149.

Van Stel, André, Martin Carree y Roy Thurik. 2005. "The Effects of Entrepreneurial Activity on National Economic Growth". Small Business Economics 24, No. 3: 311-321.

---. 2007. "The Effects of Business Regulation and Young Entrepreneurship". Small Business Economics 28, No. 2: 171-186.

Wennekers, Sanders, y otros. 2005. "Nascent Entrepreneurship and Level of Economic Development”. Small Business Economics 24, No. 3: 293-309.

Wong, Poh, Yuen Ping Ho y Erkko Autio. 2005. "Entrepreneurship, Innovation and Economic Growth: Evidence from GEM Data”. Small Business Economics 24, No. 3: 335-350. 